\title{
Where are we today? Early results from MINAP, the national audit of myocardial infarction project
}

\section{J Birkhead}

Heart 2003;89(Suppl II):ii13-ii15

The Myocardial Infarction National Audit Project (MINAP) was developed primarily as a response by the profession to the audit requirements of the National Service Framework for coronary heart disease. MINAP began to collect data in October 2000 and by October 2002, 223 hospitals in England and Wales had returned data on patients with acute myocardial infarction. MINAP provides contemporary analyses of hospital performance, with the ability to compare local performance against the national aggregate. In the third quarter of $2002,67 \%$ of patients received thrombolytic treatment within 30 minutes of hospital arrival. At the same time only $37 \%$ of patients received treatment within 60 minutes of calling for help, and only about $20 \%$ reached hospital within 30 minutes of calling for help. In order to improve speed of access to thrombolytic treatment there is a need for increased use of pre-hospital treatment.

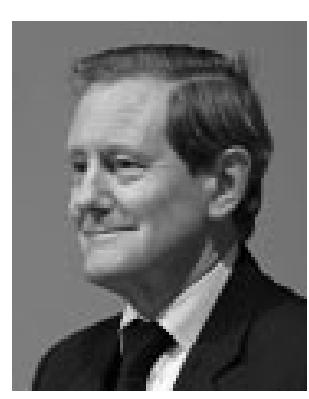

$T$ he Myocardial Infarction National Audit Project (MINAP) was established as a response by the profession to the audit requirements of the National Service Framework (NSF) for coronary heart disease. ${ }^{1}$ In developing the project it was recognised that it was important to encourage clinicians to accept ownership of clinical data in order that they would then accept the analyses of the data that were produced. The project also merges clinical audit and performance management data by providing analyses for both purposes from the same source data.

MINAP was developed by the Clinical Effectiveness and Evaluation Unit at the Royal College of Physicians of London. The project relies on a close collaboration with the Central Cardiac Audit Database (CCAD) group, based at the Royal Brompton Hospital. Using secure and encrypted transmission of data via the internet or National Health Service net, anonymised patient records are analysed centrally and comparisons of local and national aggregate performance are made available to participating hospitals on-line, and are updated every 24 hours.

CCAD is also developing links to audit data from the Society of Cardiothoracic Surgeons and the British Cardiovascular Intervention Society. As these links develop MINAP will become more than a simple audit of process and outcome of myocardial infarction, as it will then be possible to examine subsequent management of patients in terms of intervention such as coronary artery bypass surgery and angioplasty. CCAD is already linked to the Office of National Statistics allowing provision of case fatality data.

In the two years since MINAP started to collect records in October 2000, data have been collected on more than 100000 patients. Of these, about $33 \%$ had ST segment elevation acute myocardial infarction, $40 \%$ had non-ST segment elevation acute myocardial infarction, and the majority of the remainder had acute coronary syndromes. A total of 222 out of approximately 235 hospitals in England and Wales that manage acute myocardial infarction are participating in the project.

The data presented relate to hospital performance against the NSF target aimed to reduce delay in the use of thrombolytic treatment for patients with myocardial infarction. The target was that by April 2002, 75\% of eligible patients should receive thrombolytic treatment within 30 minutes of hospital arrival ("door-to-needle" time), and by April 2003 to receive thrombolytic treatment within 20 minutes of hospital arrival. In addition hospitals were required to collect audit data showing the number and percentage of patients reaching hospital within 30 minutes of a call for help and the number and percentage having treatment within 60 minutes of a call for help.

\section{IMPACT OF PATIENT DELAY CALLING FOR HELP}

There is a risk in focusing too closely on the doorto-needle interval as this tends to deflect attention from other aspects of delay from onset of patient symptoms to arrival in hospital. Potentially the greatest component of delay in the provision of treatment results from the failure by the patient with symptoms to call for help. There are many reasons why patients delay calling for help, and this delay varies considerably with the time of symptom onset, being greater when symptoms start during the night than when they start during the daytime (fig l). There is a close relation between delay by the patient in seeking help and delay to treatment with thrombolytic agents. This is important because of the close relation between the interval from onset of symptoms to provision of thrombolytic treatment and subsequent mortality. ${ }^{2}$

\section{HOW PATIENTS REACH HOSPITAL}

MINAP data for 2002 were compared with data collected from an audit carried out in 1993 and 1997 of patients with myocardial infarction in 15 hospitals in the UK. ${ }^{3}$ The earlier data, although relating to a much smaller patient sample, were

Abbreviations: CCAD, Central Cardiac Audit Database CCU, cardiac care unit; MINAP, Myocardial Infarction National Audit Project; NSF, National Service Framework 


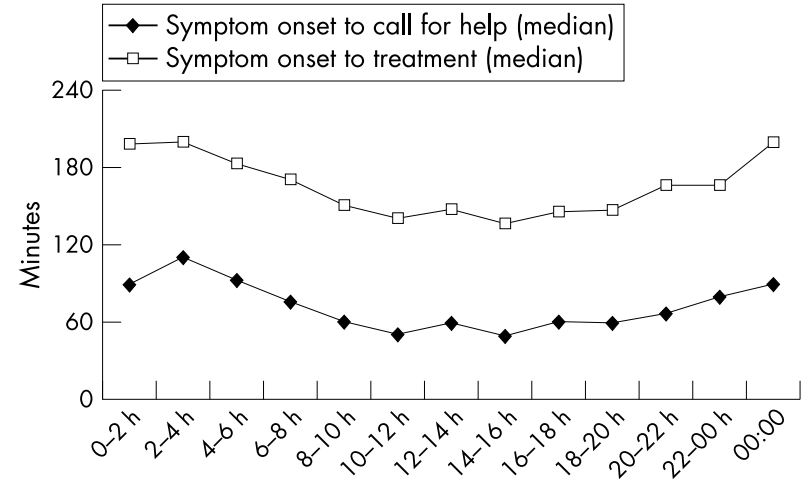

Figure 1 Impact of patient delay on time to treatment 2001-2.

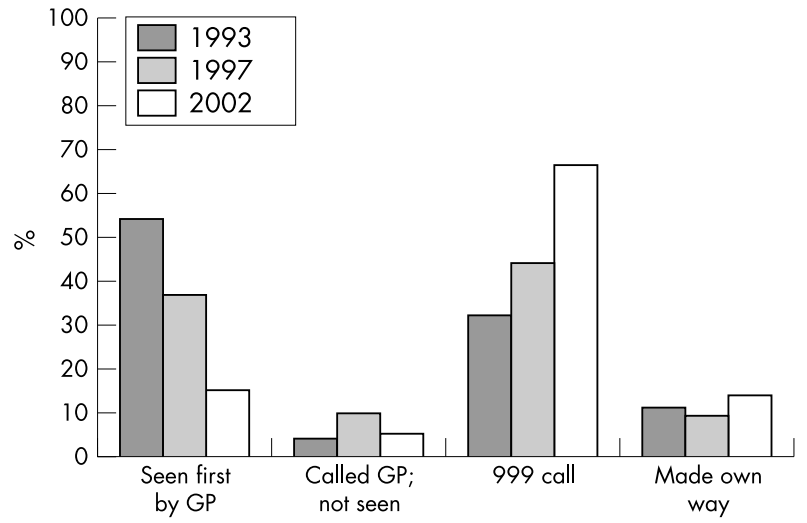

Figure 2 How patients reach hospital 1993-2002.

collected to the same criteria as the MINAP data. There has been a substantial fall in the number of patients ringing their general practitioner and an increase in direct calls to the emergency service. Calls to the general practitioner fell from $54 \%$ in 1993 to $37 \%$ in 1997 and to $15 \%$ in 2002, while calls to the emergency service increased from $32 \%$ in 1993 to $44 \%$ in 1997 and to $66 \%$ in 2002. The proportion of patients making their own way to hospital has varied only slightly, between $11 \%$ in 1993 and $14 \%$ in 2002 (fig 2). It is well recognised that patients who call 999 reach hospital more quickly than those who are seen by a general practitioner.

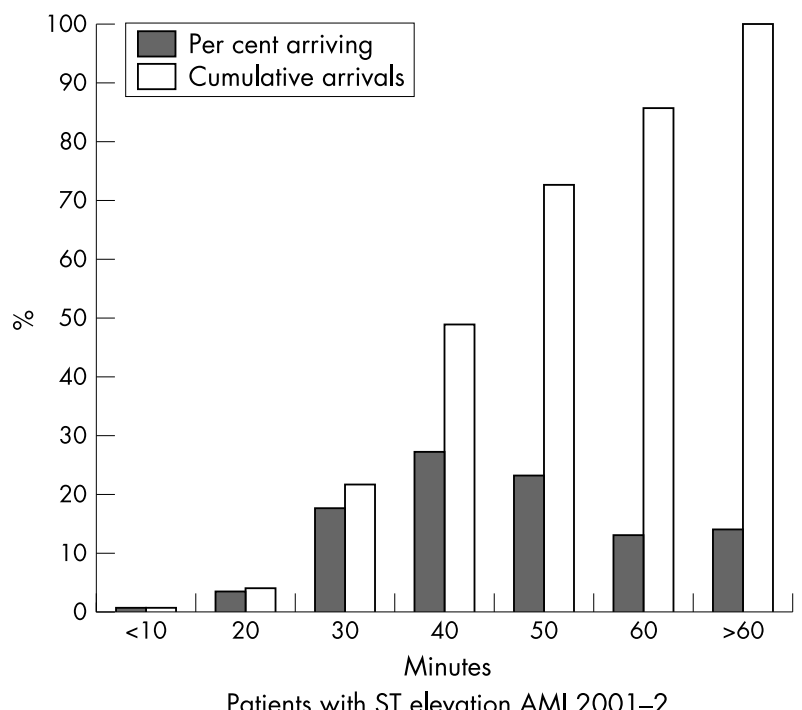

Figure 3 Reaching hospital by ambulance: interval after a 999 call for help.

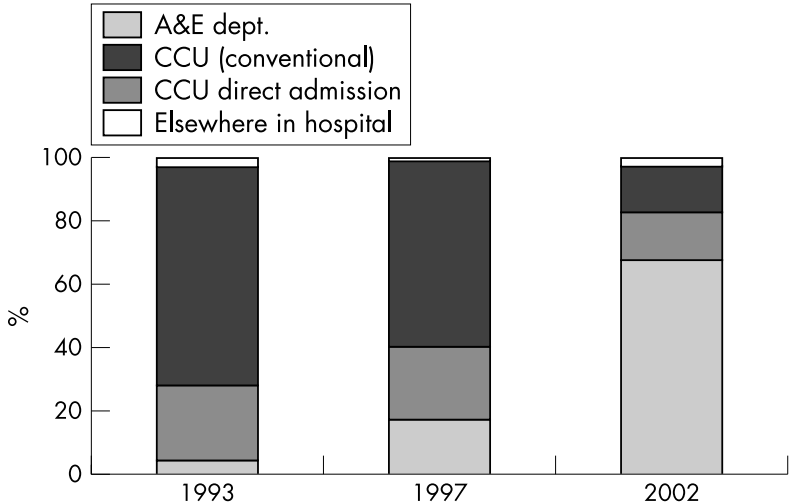

Figure 4 Increasing use of accident and emergency department for thrombolytic treatment.

Table 1 Where thrombolytic treatment is given in hospital, with median interval from arrival to treatment. Data from 2002

\begin{tabular}{lll}
\hline & Number & $\begin{array}{l}\text { Median (IQR } \\
\text { minutes }\end{array}$ \\
\hline In accident and emergency department & $7515(66.6 \%)$ & $25(17-38)$ \\
Direct admission to cardiac care unit & $1710(15.1 \%)$ & $22(15-30)$ \\
Cardiac care unit (slowtrack) & $1614(14.3 \%)$ & $44(28-69)$ \\
Elsewhere in hospital & $351(3.1 \%)$ & $27(19-47)$ \\
Not stated & $141(1 \%)$ & $29(18-46)$ \\
Overall & 11292 & $26(18-42)$
\end{tabular}

$I Q R$, interquartile range is from the 25 th to the 75 th centile.

In addition to reaching hospital more quickly, greater use of the ambulance service has the additional benefit of providing earlier access to a defibrillator, which has been shown to have significant mortality benefit; more lives may be saved by defibrillation by paramedics in an ambulance than are saved by thrombolytic treatment. ${ }^{4}$ However, the ambulance journey may be lengthy; MINAP data for 2001-2 show that $50 \%$ of patients with ST segment elevation infarction who call the ambulance service do not reach hospital within 40 minutes (fig 3). This, and the recognition that very early thrombolytic treatment carries substantial benefit, makes a strong case for use of pre-hospital thrombolytic treatment, which has now been accepted by the UK Department of Health. Agreement has been reached for the use of single bolus thrombolytic drugs by ambulance paramedic crews, and training is in progress. This development, which will be carefully evaluated by MINAP, is essential in order to make further inroads into the delay before treatment; further reductions in delay in hospital, although desirable, will be of limited overall benefit.

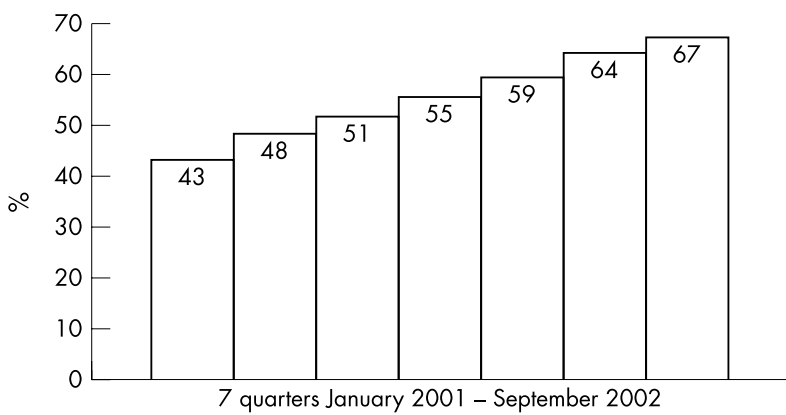

44 (21\%) hospitals reached this target in either Q4 2001 or Q1 2002 $88(42 \%)$ hospitals reached target in either Q1 or Q2, or Q3 of 2002

Figure 5 Proportion of patients having thrombolytic treatment within 30 minutes of arrival at hospital. 


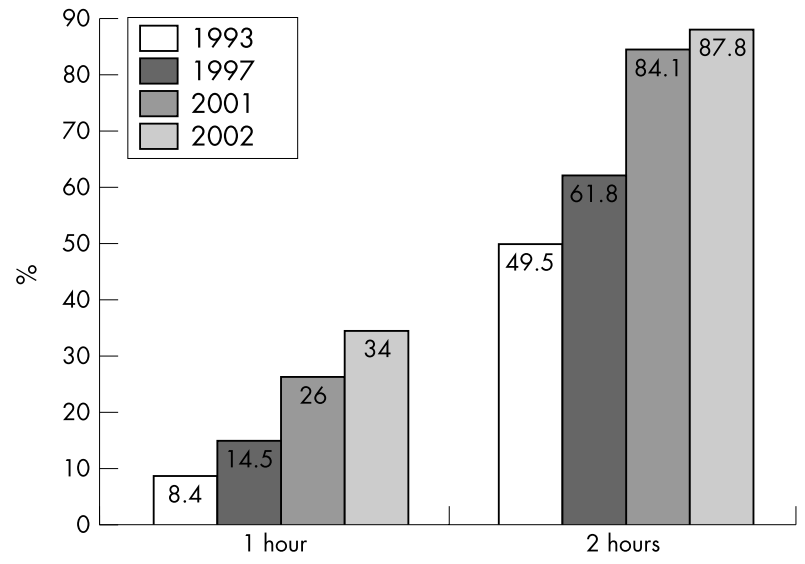

Figure 6 Use of thrombolytic treatment: intervals after a call for help.

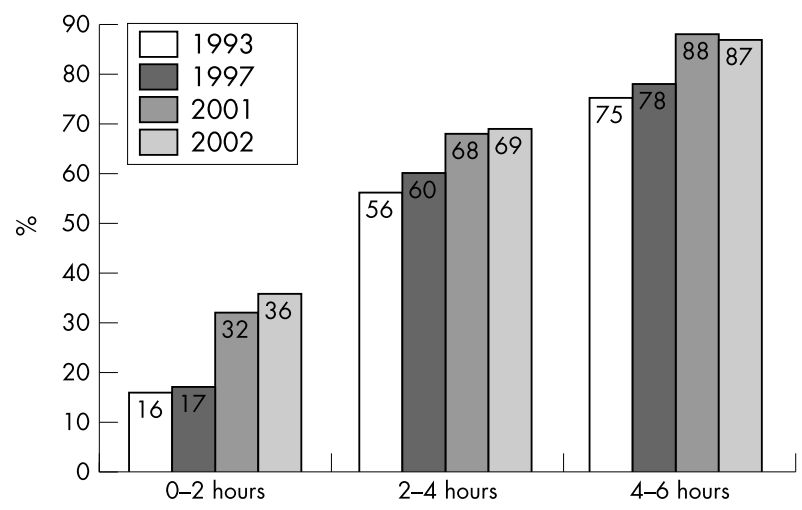

Figure 7 Use of thrombolytic treatment: intervals after onset of symptoms.

\section{WHERE TREATMENT IS GIVEN IN HOSPITAL}

There has been substantial change in where thrombolytic treatment is given in hospital since the 1993-97 audit. The proportion of patients receiving thrombolytic treatment in accident and emergency departments has increased from $4 \%$ in 1993 to $67 \%$ in 2002 (fig 4). In addition about $15 \%$ are admitted direct to the cardiac care unit (CCU) for thrombolytic treatment. Within hospital thrombolytic treatment provided after direct admission to a CCU, or in an accident and emergency department, is the most rapid, but treatment in a CCU after admission elsewhere in hospital takes substantially longer (table 1). At present more than $80 \%$ of patients receive thrombolytic treatment either after direct admission to a CCU or in accident and emergency departments. Overall the median door-to-needle time for all treatment given in hospital has halved over the past eight years, from 53 minutes in 1993 to 26 minutes in 2002 .

The proportion of patients receiving treatment within 30 minutes of arrival at hospital has increased in a linear fashion between the first quarter of 2001 (43\%) and the third quarter of 2002 (67\%) (fig 5). At the present rate of increase the NSF target will be reached during the first quarter of 2003 . However, the number of hospitals reaching the NSF target at October 2002 remains under 50\%.

\section{Learning points}

- The proportion of patients with acute myocardial infarction given thrombolytic treatment within two hours of symptom onset has doubled over the past eight years

- Future improvements are likely to come from wider use of pre-hospital thrombolysis

- Hospitals that contribute data to MINAP are able to view on-line their hospital's performance in terms of NSF targets compared with national aggregate data

- Analyses provided are contemporary, and thus of immediate value to clinicians

Overall there have been considerable improvements in hospital performance over the past eight years. Although only $34 \%$ of patients received treatment within 60 minutes of a call for help in 2002, this should be compared with 8\% in 1993 (fig 6). A more important measure of improvement is the reduction in delay from time from symptom onset to thrombolysis (fig 7); the proportion of patients receiving treatment within the first two hours has more than doubled, from $16 \%$ in 1993 to $36 \%$ in 2002 . These are the crucial hours that really matter, and this improvement represents a significant health gain.

\section{CONCLUSION}

MINAP has been developed into a powerful tool with which to examine national performance in managing myocardial infarction; early analyses show encouraging improvement. The latest data from MINAP show that just under $70 \%$ of patients received thrombolytic treatment within 30 minutes of hospital arrival, a substantial improvement over two years. However, only $37 \%$ of patients received treatment within 60 minutes of calling for help. Further improvement in this figure may have to await increasing use of pre-hospital thrombolytic treatment as there has been no real change in the interval from a call for help to reaching hospital, and further reductions in door-to-needle times will have limited impact.

\section{REFERENCES}

1 Department of Health. National service framework for coronary heart disease. London: Department of Health, 2000.

2 Boersma E, Maas ACP, Deckers JW, et al. Early thrombolytic treatment in acute myocardial infarction: reappraisal of the golden hour. Lancet 1996;348:771-5.

3 Birkhead JS, on behalf of the Myocardial Infarction Audit Group. Trends in the provision of thrombolytic treatment between 1993 and 1997. Heart 1999;82:438-42.

4 United Kingdom Heart Attack Study (UKHAS) Collaborative Group. Effect of time from onset to coming under care on fatality with acute myocardial infarction: effect of resuscitation and thrombolytic treatment. Heart 1998;80:114-20.

\section{DISCUSSION}

Question: Who do you envisage might give pre-hospital thrombolysis?

Dr Birkhead: I think it is primarily going to be the paramedics but some general practitioners in remote parts of the country are doing this. However, administration of thrombolytic treatment outside of a hospital setting is still infrequent, with only around 180 doses given so far in 2002.

Professor Lewis Ritchie: In rural Scotland, where I work, at present if GPs do not give thrombolysis then patients do not receive the treatment. 